\title{
Mapping $N$-Terminus Phosphorylation Sites and Quantitation by Stable Isotope Dimethyl Labeling
}

\author{
Chin-Jen Wu, Jue-Liang Hsu, Sheng-Yu Huang, and Shu-Hui Chen \\ Department of Chemistry, National Cheng Kung University, Tainan, Taiwan
}

\begin{abstract}
We have previously coupled stable isotope dimethyl labeling with IMAC enrichment for quantifying the extent of protein phosphorylation in vivo. The enhanced $a_{1}$ signal of dimethylated peptides served as a unique mass tag for unequivocal identification of the $\mathrm{N}$-terminal amino acids. In this study, we demonstrate that the $\mathrm{a}_{1}$ ion could further assist in mapping the precise phosphorylation site near the $\mathrm{N}$-terminal region and allow the determination of the exact site and level of phosphorylation in one step by stable isotope dimethyl labeling. We show that the $\mathrm{a}_{1}$ ion signal was suppressed for dimethylated peptides with a phosphorylation site at the $N$-terminus Ser/Thr residue (N-p*Ser/Thr) but was still enhanced for $N$-terminus Tyr residue ( $N$ - $\left.{ }^{*} T y r\right)$ or internal Ser/Thr residues (-p*Ser/Thr). Based on the dominant de-phosphorylated molecular ions and b- $\mathrm{H}_{3} \mathrm{PO}_{4}$ ions for $\mathrm{N}-\mathrm{p}$ * $\mathrm{Ser} / \mathrm{Thr}$, we propose that dimethyl labeling increases the basicity of the N-terminus and accelerates the dephosphorylation for N-p*Ser/Thr precursors, which, however, suppresses the $a_{1}$ ion enhancement due to the resulting unsaturated covalent bond on $\mathrm{C}_{\alpha}$ of the $\mathrm{N}$-terminus amino acid. Using this method, we excluded three Ser/Thr phosphorylation sites in A431 cells, two of which, however, were previously reported to be phosphorylation sites; we confirmed three known phosphorylation sites in A431 cells and quantified their ratios upon EGF treatment. Notably, we identified a novel phosphorylation site on Ser43 residue at $N$-terminus of the tryptic peptide derived from SVH protein in pregnant rat uteri. SVH protein has not been reported or implied with any phosphorylation event, and our data show that the Ser43 of SVH is an intrinsic phosphorylation site in pregnant rat uteri and that its phosphorylation level was slightly decreased upon c-AMP treatment. (J Am Soc Mass Spectrom 2010, 21, 460-471) (C 2010 American Society for Mass Spectrometry
\end{abstract}

$\mathrm{R}$ eversible protein phosphorylation is crucial for many regulatory and functional features of living organisms such as intercellular communication, cell growth, proliferation, differentiation, and apoptosis [1-4]. For proteins containing multiple phosphorylation sites, each phosphorylation site could be regulated by different kinases and involved in diverse signal transduction pathways in eukaryotic cells. Thus, identification of the exact site and the extent of phosphorylation are crucial for understanding the role of dynamic protein phosphorylation. Traditionally, protein phosphorylation sites are determined by Edman sequencing coupled with ${ }^{32} \mathrm{P}$ labeling [5-7]. The use of radioisotopes provides a sensitive detection method, but it also suffers from the hazard of radioactivity $[8,9]$. In recent years, tandem mass spectrometry (MS/MS) integrated with database searches or de novo sequencing for protein identification has become an excellent tool. Coupled with various enrichment techniques, such as immobilized metal affinity chromatography (IMAC)

Address reprint requests to Dr. S.-H. Chen, Department of Chemistry, National Cheng Kung University, No. 1 College Road, Tainan 701, Taiwan. E-mail: shchen@mail.ncku.edu.tw or $\mathrm{TiO}_{2}$ and stable isotope labeling techniques, the MS-based approach is becoming promising for qualitative and quantitative analyses of phosphoproteins. However, many challenges still remain for MS-based phosphoproteomics because peptides are phosphorylated at sub-stoichiometric levels. At such low amounts, signal suppression effects by non-phosphopeptides and difficulties in achieving full coverage of long tryptic peptides hinder MS-based phosphoproteomic analysis [10-13]. In addition, post-translation modification is not included in the genome database, thus hampering the identification of exact phosphorylation sites by database assisted methods. Determination of exact phosphorylation sites among multiple possible phosphorylation sites within a given peptide fragment is difficult under collision induced dissociation (CID) of the MS/MS mode due to incomplete fragmentation and partial dephosphorylation. Such ambiguous assignments are more serious for potential sites centralized in either $\mathrm{N}$ - or $\mathrm{C}$-terminal since they are more clearly identified by same types of fragments, $\mathrm{b}$ and $\mathrm{y}$ ions, respectively. Although electron-transfer dissociation (ETD) [14-16] could provide better backbone fragmentation than the CID method, ETD has limitations for 
peptides with low charge state. Moreover, CID is still far more robust and more frequently used for protein identification.

Many noteworthy chemical approaches have been developed to assist in identifying protein phosphorylation sites. For instance, Knight et al. used $\beta$-elimination of phosphate groups with a barium catalyst in sodium hydroxide followed by Michael addition with cysteamine to convert phosphoserine and phosphothreonine into lysine analogues, which could be recognized and cleaved with endoproteinase Lys-C. Due to the diastereomeric aminoethylcysteine $(R / S)$ peptide mixture generated by the reaction, cleavages only occur on the $\mathrm{R}$ isomeric form of the lysine analogue. Such partial cleavage creates a ladder of peptides consisting of successive single cleavage and, together with the unique $\mathrm{y}_{1}$ mass tag associated with the converted lysine analogue, can be used to assist in identifying protein phosphorylation sites [17, 18]. Kristensen et al. also reported a strategy using uranyl (VI) photocleavage to recruit the phosphopeptides by affinity interaction between uranyl ion and the phosphate group and then cleave at $N$-proximal to the phosphorylated residues by irradiation at $320 \mathrm{~nm}$. The phosphorylation site could then be identified by MS via such site specific cleavage [19]. There are, however, one or two drawbacks associated with these methods, including complex and laborious processes and long cleavage fragments that are hard to analyze by MS.

We have previously coupled stable isotope dimethyl labeling with IMAC enrichment for quantifying the extent of protein phosphorylation in rat uteri, and the enhanced $\mathrm{a}_{1}$ signal of dimethylated peptides serves as a unique mass tag for unequivocal identification of $N$ terminal amino acids [20, 21]. Compared with other labeling methods, this labeling reaction is fast (less than $5 \mathrm{~min}$ ) and does not produce any detectable byproducts. In this study, we further explore how the $a_{1}$ ion could assist in mapping the precise site of phosphorylation at $\mathrm{N}$-terminus Ser/Thr and Tyr residue and allow the determination of the exact site and the level of phosphorylation in one step by stable isotope dimethyl labeling. We will characterize the fragmentation by standard phosphopeptides and then demonstrate the usefulness of the method by qualitatively and quantitatively analyzing phosphoproteins stimulated by MARK and PKA kinases in cells and tissues. Based on the observations, we have proposed fragmentation pathways that could explain the results.

\section{Experimental}

\section{Materials}

Acetonitrile, ammonium hydroxide, acetic acid, and formaldehyde $\left(37 \%\right.$ in $\left.\mathrm{H}_{2} \mathrm{O}\right)$ were purchased from J. T. Baker (Phillipsburg, NJ, USA). Trichloroacetic acid (TCA), formic acid (98\% 100\%), iron(III) chloride, so- dium acetate, ammonium bicarbonate, and sodium chloride were obtained from Riedel-de Haven (Seelze, Germany). D,L-dithiothreitol (DTT), sodium cyanoborohydride. The synthetic peptides, SSpSPAPADIAQTVQEDLR and pSSSPAPADIAQTVQEDLR were purchased from Sigma (St. Louis, MO, USA); pTQTPPVSPAPQPTEER and pYLLNQGADVTLR were synthesized by MDBio (Taipei, Taiwan). Formaldehyde- $D_{2}$ was obtained from Aldrich (Milwaukee, WI, USA). Iodoacetamide (IAM) and trypsin were purchased from Fluka (Buchs, Switzerland) and Promega (Madison, WI, USA), respectively. The water was from an E-pure water purification system (Barnstead Thermolyne Corp., Dubuque, IA, USA).

\section{Cell Culture and Rat Uterus Homogenate}

A431 cells were cultured in Dulbecco's modified Eagle's medium (Sigma-Aldrich, Louis, MO. USA) supplemented with $10 \%$ fetal bovine serum, penicillin/ streptomycin (Gibco/Invitrogen, Carlsbad, CA, USA) and $5 \% \mathrm{CO}_{2}$ at $37^{\circ} \mathrm{C}$. Cells were cultured for an additional $24 \mathrm{~h}$ in the absence of serum and then treated with $50 \mathrm{ng} / \mathrm{mL}$ of EGF (Upstate Biotechnology, Waltham, MA, USA) for $20 \mathrm{~min}$ at $37^{\circ} \mathrm{C}$. After the treatment, cells were washed two times with ice-cold PBS and then were lysed in modified RIPA buffer containing $50 \mathrm{mM}$ Tris- $\mathrm{HCl}, 1 \% \mathrm{NP} 40,0.25 \%$ sodium deoxycholate, $150 \mathrm{mM} \mathrm{NaCl}, 1 \mathrm{mM}$ EGTA, $1 \mathrm{mM}$ PMSF, $1 \mu \mathrm{g} / \mathrm{mL}$ aprotinin, $1 \mu \mathrm{g} / \mathrm{mL}$ leupeptin, $1 \mu \mathrm{g} / \mathrm{mL}$ pepstatin, $1 \mathrm{mM} \mathrm{NaF}$, and $1 \mathrm{mM}$ sodium orthovanadate at $\mathrm{pH}$ 7.4. Uterus homogenate of female Wistar rats (ca. 200-350 g) sacrificed on gestation d 18 was prepared and analyzed as described in a previous study [22].

\section{IMAC and Dimethyl Labeling}

Cell lysate (500 $\mu \mathrm{g}$ total protein) or uterus homogenate (1 mg total protein) was reduced with $10 \mathrm{mM}$ DTT at $95^{\circ} \mathrm{C}$ for $5 \mathrm{~min}$. The sample was then alkylated with 50 mM IAM (A431 cells) or IAA (uterus homogenate) in the dark at room temperature for $30 \mathrm{~min}$ and then concentrated by TCA precipitation. After washing with $10 \%$ TCA and deionized water, $4 \mu \mathrm{g}$ trypsin dissolved in $200 \mu \mathrm{L}$ of $50 \mathrm{mM}$ ammonium bicarbonate ( $\mathrm{pH} 7.8)$ was added and left to react at $37^{\circ} \mathrm{C}$ for $18 \mathrm{~h}$. A slurry of POROS MC20 (Applied Biosystems, Foster city, CA, USA) in deionized water was packed under vacuum into a $31.6 \mu \mathrm{L}$ cartridge. After equilibration with $0.1 \%$ acetic acid, the packed cartridge was filled with $25 \mathrm{mM}$ iron (III) chloride in $0.75 \mathrm{M}$ sodium acetate, and was then reconditioned with $0.1 \%$ acetic acid before use. The tryptic digests were loaded into the prepared IMAC cartridge, washed with two buffers in sequence, the buffer containing $0.1 \%$ acetic acid and $30 \% \mathrm{ACN}$, and then the buffer containing $0.1 \%$ acetic acid for a total of six cartridge volumes. The digests were then eluted with a buffer containing $0.5 \%$ ammonium hydroxide ( $\mathrm{pH}$ 9.5). The eluted fractions were dried under vacuum. The phos- 
phopeptides purified by IMAC or standard phosphopeptides (100 pmol) were diluted with $100 \mu \mathrm{L}$ of sodium acetate buffer $(100 \mathrm{mM}, \mathrm{pH}$ 5.6). The solution was then mixed with formaldehyde ( $4 \%$ in water, $4 \mu \mathrm{L}$ ), vortexed, and then mixed immediately with freshly prepared sodium cyanoborohydride $(600 \mathrm{mM}, 4 \mu \mathrm{L})$. The mixture was vortexed again and then allowed to react for $5 \mathrm{~min}$. Finally, ammonium hydroxide ( $4 \%$ in water, $4 \mu \mathrm{L}$ ) was added to consume the excess formaldehyde. After desalting and drying, the samples were redissolved in a buffer containing $5 \%$ acetonitrile and $0.1 \%$ formic acid for LC/ MS/MS analysis.

\section{Instrumentation}

For labeling efficiency, the MS data were obtained using a MALDI-TOF spectrometer equipped with a 337-nm $\mathrm{N}_{2}$ laser (MALDI, Micromass, Manchester, U.K.). The MALDI matrix was prepared by dissolving 4-cyanohydroxysuccinic acid (10 mg) in EtOH/MeCN $(1: 1,1 \mathrm{~mL})$ containing $0.1 \%$ TFA. A $0.5 \mathrm{M}$ solution of $\mathrm{HCl}$ was mixed with both the matrix and the $400 \mathrm{fmol} / \mu \mathrm{L}$ phosphopeptides at a ratio of matrix/HCl/sample of 2:1:1 ( $\mathrm{vol} / \mathrm{vol} / \mathrm{vol})$. The mixture was deposited on the target and dried before detection. For protein identification by peptide sequencing, the samples were analyzed by a Q-TOF micro spectrometer (Micromass, Manchester, UK) by direct infusion at a flow rate of $0.3 \mu \mathrm{L} / \mathrm{min}$ or through an on-line nanoflow HPLC system (LC Packings, Amsterdam, The Netherlands). Briefly, a volume of $25 \mu \mathrm{L}$ tryptic digest solution was injected, concentrated by a C18 nanoprecolumn cartridge (i.d. $300 \mu \mathrm{m} \times$ 1-mm, 5- $\mu \mathrm{m} \mathrm{C18,} \mathrm{P/N160458,} \mathrm{LC} \mathrm{Packings),} \mathrm{and} \mathrm{then}$ separated by a C18 column (i.d. $75 \mu \mathrm{m}$, o.d. $280 \mu \mathrm{m} \times$ 15-cm, 3- $\mu \mathrm{m}$ C18, LC Packings). Mobile phase A consisted of $0.1 \%$ formic acid in $5 \%$ acetonitrile solution, and mobile phase B consisted of $0.1 \%$ formic acid in $80 \%$ acetonitrile solution; a linear gradient from $5 \%$ to $90 \%$ B over a $60 \mathrm{~min}$ period at a flow rate of $250 \mathrm{~nL} / \mathrm{min}$ was applied. The parameters set on the Q-TOF micro instrument were as follows: capillary voltage $(3000 \mathrm{~V})$, sample cone $(60 \mathrm{~V})$, excitation cone $(0.5 \mathrm{~V})$, source temperature $\left(80^{\circ} \mathrm{C}\right)$, and collision energy $(30-50 \mathrm{~V})$. The MassLynx 4.0 Global ProteinLynx was used to produce a peak list from raw data. The MS/MS spectra were obtained by performing survey scans; the survey scan was from $\mathrm{m} / \mathrm{z} 400$ to 1600 , and MS/MS scan was from $m / z 50$ to 2000. The threshold to switch from MS to MS/MS was 10 counts.

\section{Database Search and Quantification}

Proteins were identified using the in-house MASCOT v2.2.1 search engine on the Swiss-Prot 51.6 (human) protein database ( 257,964 sequences; 15,720 human protein sequence entries) and NCBInr 20060829 (Rattus) database (3888187 sequences; 40,015 protein sequence entries) as well as on the random protein database for validation. The mass tolerance was set to be $0.2 \mathrm{Da}$ for precursor and $0.2 \mathrm{Da}$ for product ions. Phos(ST), phos $(\mathrm{Y})$, and oxidation $(\mathrm{M})$ were chosen for variable modifications; carboxymethyl- (IAA for uterus homogenate) or carbamidomethyl (IAM for A431 cells) were chosen for fixed modifications, and one trypsin miscleavage was allowed. For labeled peptides, dimethyl (heavy or light) at $\mathrm{N}$-terminal or lysine (k) was added as the variable modification. We set a score cutoff at 20 to eliminate low-score peptides, and only "rank1" (best match for each MS/MS) peptides were included. Only proteins within the significant hit lists $(P<0.05)$ were regarded as identified proteins. Under these criteria, the cut-off scores are 40 and 20 for proteins and peptides, respectively. Random database search for false identification rate was determined to be less than $3.08 \%$. Moreover, the MS/MS spectra of individual phosphopeptide ion that reaches a score beyond significant hit $(P<0.05)$ were manually inspected, and their MS/MS spectra must exhibit de-phosphate molecular ion (-98 Da) to be counted as identified. Details about phosphoproteins identified from A431 cells are listed in Table S1 of the supplementary material, which can be found in the electronic version of this article. All of the spectra containing both mass peaks of $D_{4}$ - and $H_{4}$-labeled peptides were combined to produce a composite MS spectrum. The ratios of the $D_{4^{-}}$and $\mathrm{H}_{4}$-labeled peptides in the composite MS spectra were calculated from the sum of the peak heights of the first three isotopic peaks.

\section{Results and Discussions}

\section{Labeling Efficiency for Phosphopeptides}

Whether or not the $\mathrm{N}$-terminus phosphorylation affects the labeling reaction was investigated first since dimethyl labeling occurs on both the $\mathrm{N}$-terminus and $\varepsilon$-amino group of Lys residues via reductive amination. The spectra obtained from the $H_{4^{-}}$and $D_{4}$-labeled, as well as from the unlabeled $N$-terminus phosphopeptide, pSSSPAPADIAQTVQEDLR sequence derived from Ras-GTPase-activating protein-binding protein 1 , are shown in Figure 1. The spectra indicate that the $\mathrm{m} / \mathrm{z}$ value of the unlabeled ion (Figure 1a) is 28 mass units lower relative to those of the $\mathrm{H}_{4}$-labeled ion (Figure $1 \mathrm{~b}$ ), which is consistent with the prediction made by counting a 28-mass-unit difference for each labeled site and indicates that this labeling reaction is not affected by $\mathrm{N}$-terminus phosphorylation. Moreover, since no unlabeled peptides were detected after the reaction, the labeling efficiency of phosphopeptides is near 100\% as reported for non-phosphopeptides [23] as well as for tryptic peptides with internal phosphorylation sites [22]. Figure $1 \mathrm{c}$ shows that the $D_{4}$-labeled ions are 32 and 4 mass units higher relative to the unlabeled and $\mathrm{H}_{4}$-labeled ions, respectively, which is also consistent with the prediction. The peaks at around $\mathrm{m} / \mathrm{z} 1986$ (Figure 1b) and 1990 (Figure 1c) were due to the labeled 
(a)
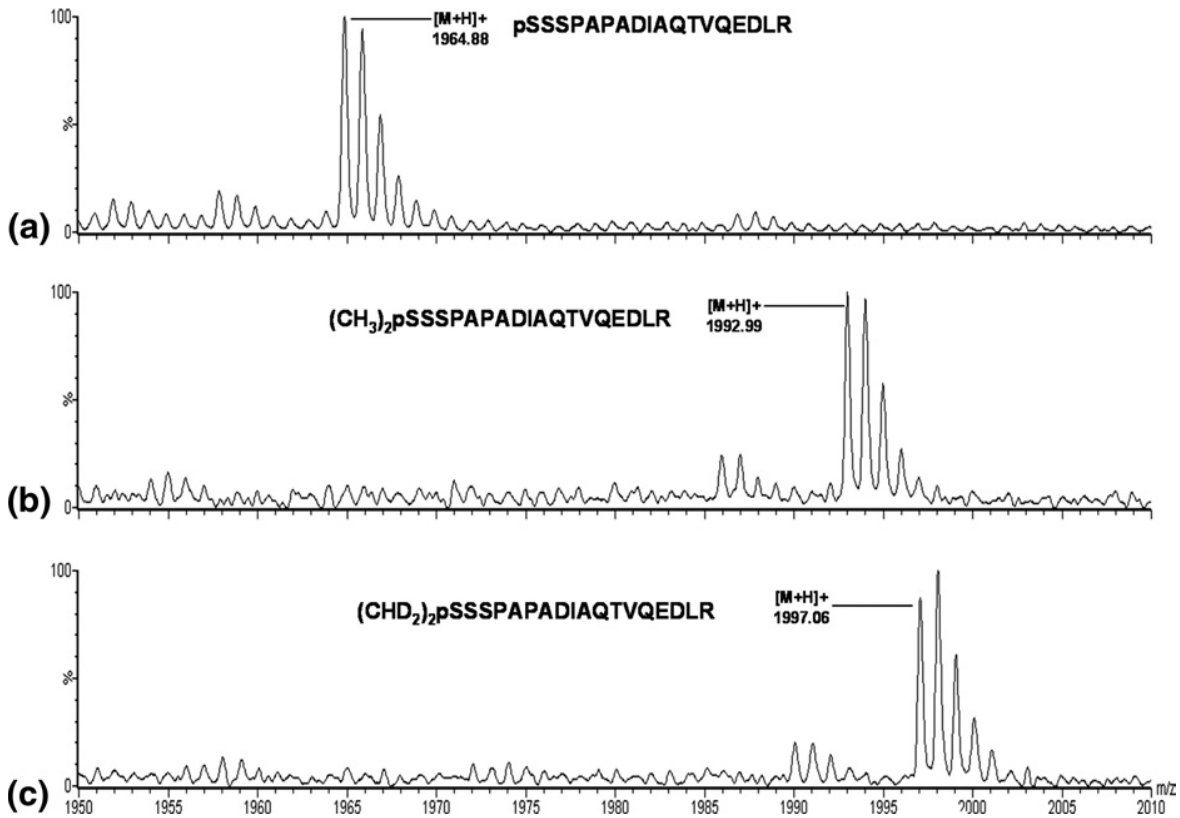

Figure 1. MALDI spectra of (a) unlabeled $N$-terminus phosphopeptide, pSSSPAPADIAQTVQEDLR derived from Ras-GTPase-activating protein-binding protein 1 , (b) labeled with $\mathrm{H}_{4}$-formaldehyde, and (c) labeled with $D_{4}$-formaldehyde.

impurities present in the synthetic phosphopeptide standard, and their unlabeled ion was indicated in Figure 1a as impurity peaks at around $m / z$ 1958. It is also worth noting that the phosphate group was not modified or cleaved by dimethyl labeling, indicating that this labeling strategy is applicable for peptides with $\mathrm{N}$-terminus phosphorylation sites.

\section{MS/MS Spectra for Unlabeled and Dimethylated Phosphopeptides}

Figure 2 displays the MS/MS spectra of the synthetic unlabeled phosphopeptides with phosphorylation at the $\mathrm{N}$ terminus Ser residue ( $N$-pSer), pSSSPAPADIAQTVQEDLR (Figure 2a) as well as at the third Ser residue (-pSer), SSPSPAPADIAQTVQEDLR (Figure 2b). Both of the precursor ions are doubly charged, and the N-pSer sequence appears to have more dephosphorylated molecular ion $\left[\mathrm{MH}_{2}-\mathrm{H}_{3} \mathrm{PO}_{4}\right]^{2+}$ relative to the -pSer sequence. In contrast, the -pSer sequence appears to have more intact molecular ion $\left[\mathrm{MH}_{2}\right]^{2+}$ relative to the $\mathrm{N}$-pSer sequence. The above results could indicate a higher probability of de-phosphorylation for $\mathrm{N}$-pSer than for - pSer and worth further investigations. Moreover, as shown in Figure 2a, the fragmentation pattern of the N-pSer peptide and -pSer peptide appears to be different. Besides the $b_{2}$ ion, there is no $b_{1}$ or $y$ ion to confirm the exact phosphorylation site among the first two residues. However, based on the $b_{x}-y_{z}$ low-energy pathway, the lack of b1 ion is expected due to a nucleophilic attack by the oxygen of the $N$-terminus amide on the second carbon center of the protonated amide (pathway B of Scheme 1). Likewise, as shown in
Figure $2 b$, there is $\left[b_{3}-\mathrm{H}_{3} \mathrm{PO}_{4}\right]^{+}$ion for -pSer, but there is no $b_{2}$ ion to confirm the exact phosphorylation site among the first three residues.

The MS/MS spectra of dimethylated phosphopeptides with phosphorylation sites at the $\mathrm{N}$-terminus $\left({ }^{*} N\right.$-pSer/Thr) and internal residues $\left({ }^{*}\right.$-pSer/Thr) were also investigated. Stable isotope dimethyl labeling is reported to provide a signal enhancement for the produced $\mathrm{a}_{1}$ or $\mathrm{y}_{\mathrm{n}-1}$ ions, which are usually hard to detect among most of the nonderivatized fragments. With a phosphorylation site at the $\mathrm{N}$-terminus $\left({ }^{*} \mathrm{~N}\right.$-pSer), the $\mathrm{a}_{1}$ signal enhancement was not observed, and with phosphorylation sites at internal residues $\left({ }^{*}\right.$-pSer), the $a_{1}$ fragment of the *-pSer ion was substantially enhanced, as shown in Figure 3. Likewise, same phenomena were also observed for peptides labeled with $D_{4}$ formaldehyde (Figure S1A and B, supplementary material). Moreover, it is worth noting that only the de-phosphorylated molecular ion $\left[\mathrm{MH}_{2}-\mathrm{H}_{3} \mathrm{PO}_{4}\right]^{2+}$ but no intact molecular ion $\left[\mathrm{MH}_{2}\right]^{2+}$ was detected for ${ }^{*} \mathrm{~N}$-pSer; in contrast, only the intact molecular ion $\left[\mathrm{MH}_{2}\right]^{2+}$ but no de-phosphorylated molecular ion $\left[\mathrm{MH}_{2}-\mathrm{H}_{3} \mathrm{PO}_{4}\right]^{2+}$ was detected for ${ }^{*}$-pSer (Figure $3 b$ ). In addition, all detected $b$ ions were dephosphorylated for ${ }^{*} N$-pSer, but there were still substantial phosphorylate b ions for * ${ }^{*}$ pSer peptides. Such results indicate that dimethyl labeling could enhance de-phosphorylation at $\mathrm{N}$-terminus sites. In addition to serine, similar results were also observed for threonine residue with stable isotope dimethyl labeling using $\mathrm{H}_{4}$ (Figure $3 \mathrm{c}$ ) and $D_{4}$ (Figure S2, supplementary material) formaldehyde. As displayed in the spectra, the $a_{1}$ ion of ${ }^{*}$ PTQTPPVSPAPQPTEER ( $\left.{ }^{*} N-p T h r\right)$ was lacking, and only the dephosphorylated molecular ion $\left[\mathrm{MH}_{2}\right.$ 

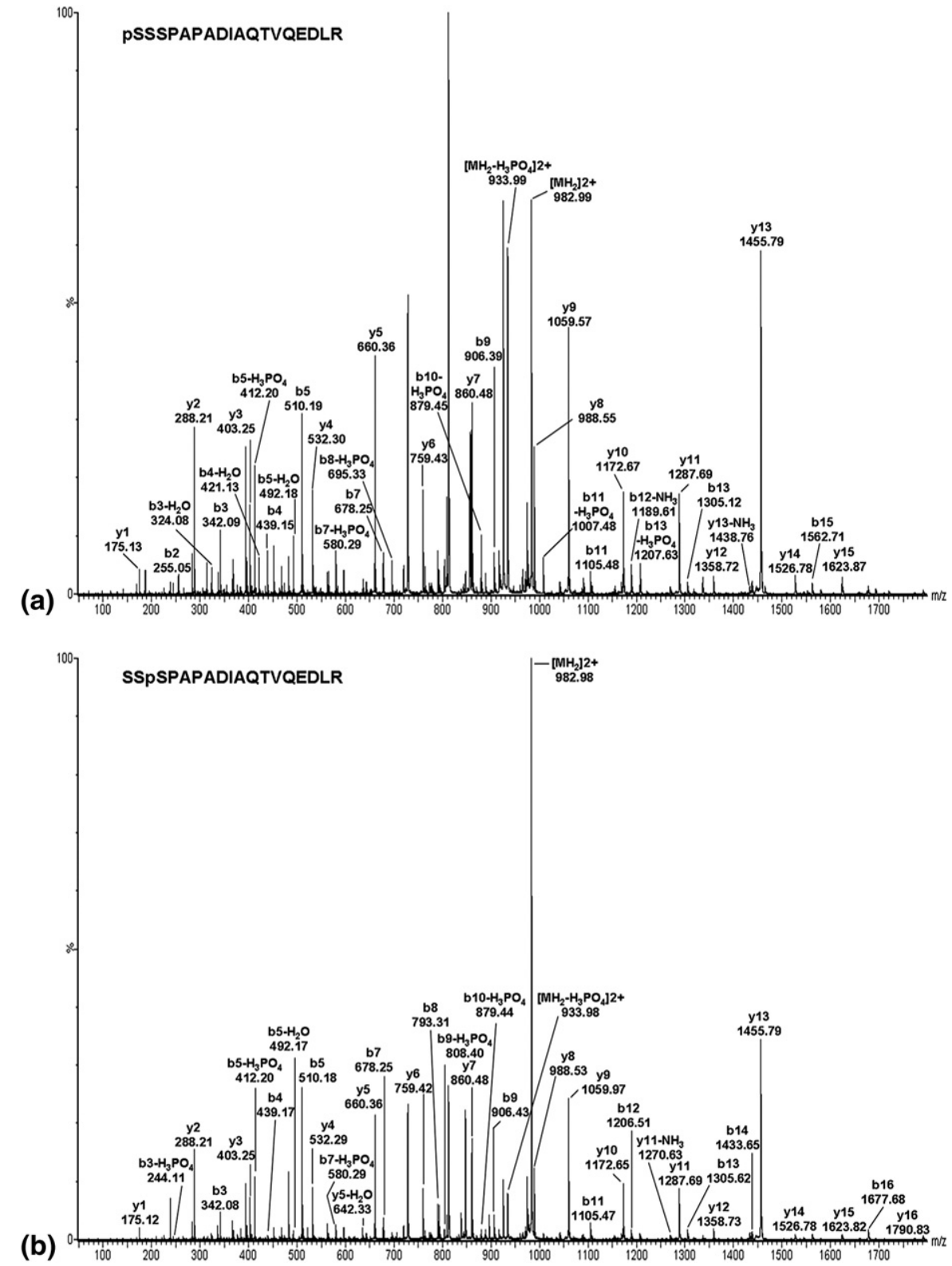

Figure 2. MS/MS spectra of the synthetic phosphopeptides with phosphorylation at (a) Nterminus Ser residue (N-pSer), pSSSPAPADIAQTVQEDLR and (b) at the third Ser residue (-pSer), SSPSPAPADIAQTVQEDLR.

$\left.\mathrm{H}_{3} \mathrm{PO}_{4}\right]^{2+}$ was detected. We have also examined the MS/MS spectra of a dimethylated peptide containing phospho-tyrosine at $N$-terminus, *pYLLNQGADVTLR ( ${ }^{*} \mathrm{~N}$-pTyr) using $\mathrm{H}_{4}$ (Figure $3 \mathrm{~d}$ ) and $\mathrm{D}_{4}$ (Figure S3, supplementary material) formaldehyde for the labeling. However, as shown in the spectra, no de-phosphorylated molecular ion $\left[\mathrm{MH}_{2}-\mathrm{H}_{3} \mathrm{PO}_{4}\right]^{2+}$ was detected, and the phosphotyrosine at the $N$-terminus can be unambiguously assigned by the enhanced $\mathrm{a}_{1}$ ion with $\mathrm{m} / \mathrm{z} 244 \mathrm{Da}$.

In addition to the above three (S/T/Y) sequences, we have investigated other MS/MS spectra acquired from a large scale phosphoproteomics analysis (data not shown).
We found no exceptions of MS/MS spectra derived from $N$-terminus phosphopeptides (11 cases) and from non- $N$ terminus phosphopeptides against the conclusions we made. Three additional representative MS/MS spectra derived from $N$-terminus phosphopeptides were shown in Figure S4-S6 (supplementary material). Among them, the spectra derived from ${ }^{*} N$-pSer [ $\left(\mathrm{CH}_{3}\right)_{2}$ pSPGMLEPLGSSR] (Figure S4) and ${ }^{*} \mathrm{~N}$-pSer $\left[\left(\mathrm{CH}_{3}\right)_{2}\right.$ pSGEGEVSGLMR] (Figure S5) were lack of $\mathrm{a}_{1}$ ion and the dephosphorylated molecular/ fragment ions predominantly occurred. On the other hand, the spectrum of ${ }^{*} \mathrm{~N}$-pTyr $\left[\left(\mathrm{CH}_{3}\right)_{2} \mathrm{pYLYEIAR}\right]$ (Figure S6) exhibits the enhanced $\mathrm{a}_{1}$ ion and the intact molecular/ 


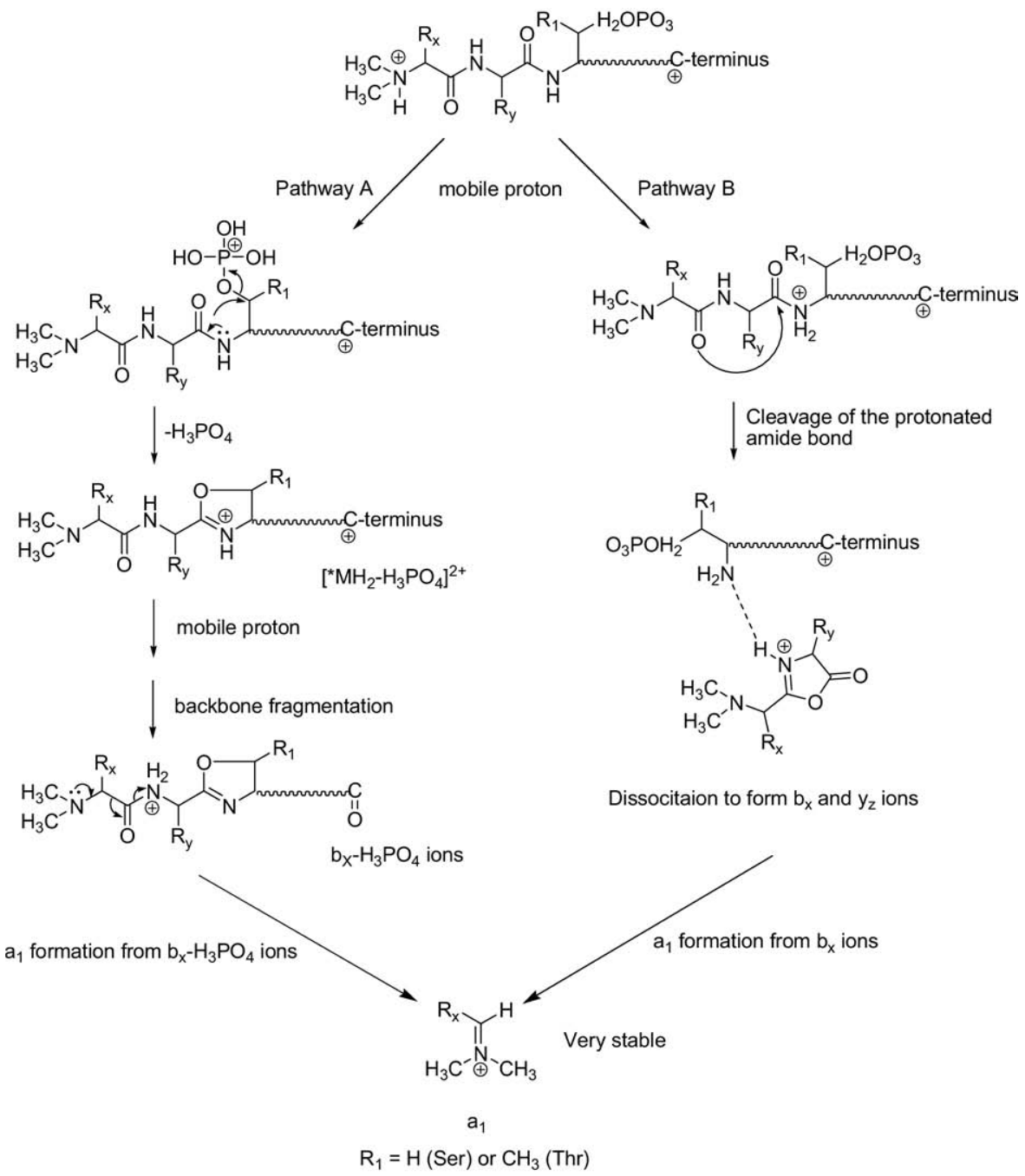

Scheme 1. Fragmentation pathways for dimethylated phosphopeptides with phosphorylation sites at the internal residues $\left({ }^{*}\right.$-pSer/Thr).

fragment ions predominantly occurred. Thus, we believe our conclusion is valid.

\section{Fragmentation Pathway}

Our results clearly indicate that dimethyl labeling leads to enhanced dephosphorylated molecular $\left[\mathrm{MH}_{2}-\right.$ $\left.\mathrm{H}_{3} \mathrm{PO}_{4}\right]^{2+}$ and $\left[\mathrm{b}-\mathrm{H}_{3} \mathrm{PO}_{4}\right]^{+}$ions derived from ${ }^{*} \mathrm{~N}$ pSer/Thr precursors but not for either ${ }^{*}$-pSer/Thr or ${ }^{*} N$-pTyr precursors. Possible mechanisms are proposed as depicted in Schemes $\mathbf{1}$ and $\mathbf{2}$ to explain these observations. By protonation, a tryptic peptide precursor normally is doubly charged. The charge commonly resides on the $\mathrm{N}$-terminus and the basic $\mathrm{R}$ or $\mathrm{K}$ residue at the $\mathrm{C}$-terminus initially. Dimethyl labeling will increase the basicity of the $N$-terminus and thus further increase the chance of $N$-terminus protonation. For *-pSer/Thr precursor, as shown in Scheme 1, the fragmentation occurs as protons mobilize from the $N$-terminus to the phosphate group (pathway A) or to the $N$ atom along the main chain (pathway B). The former will lead to dephosphorylation of the molecular ion via a charge-directed $\mathrm{SN}_{2}$ neighboring group participation reaction or a charge-directed E2 elimination reaction [24, 25], which could subsequently undergo a sequence of backbone cleavages; the later will directly lead to backbone cleavages. The b ions created by backbone cleavages either via pathways A or B are thus composed of both dephosphorylated and phosphorylated $b$ ions, both of which could lead to stable $a_{1}$ ions via concerted cleavages at the $\mathrm{C}_{\alpha}-\mathrm{C}_{\text {amide }}$ bonds, as we and other group had reported previously $[20,26,27]$.

For ${ }^{*} N$-pSer/Thr precursor, we think the increased basicity of the $N$-terminus by dimethyl labeling will substantially diminish the mobility of the charged $\mathrm{N}$ terminus proton. On the other hand, we think that the protonated $N$-terminus will stabilize the product of $\beta$-elimination, which occurs between the adjacent $C_{\alpha}$ and $C_{\beta}$ by inductive effect and thus enhance the dephosphor- 

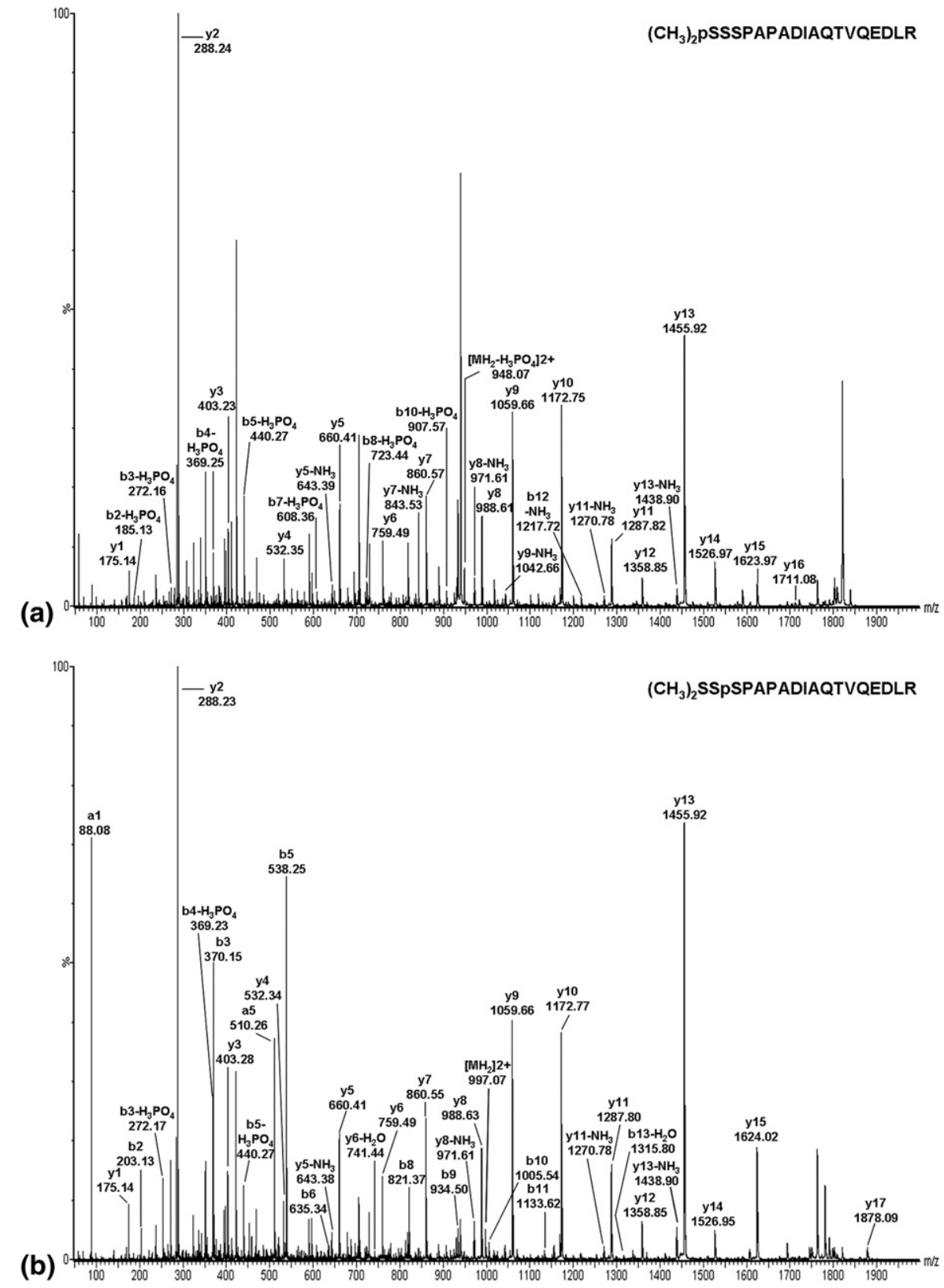

Figure 3. MS/MS spectra of dimethylated phosphopeptides with a Ser phosphorylation site at (a) $\mathrm{N}$-terminus, *pSSSPAPADIAQTVQEDLR and $(\mathbf{b})$ at internal residue, *SSpSPAPADIAQTVQEDLR; with (c) Thr phosphorylation site at $N$-terminus, *pTQTPPVSPAPQPTEER, and with (d) Tyr phosphorylation site at $N$-terminus, ${ }^{*}$ PYLLNQGADVTLR.

ylation of the $N$-terminus phosphorylated precursor ion. Taken together, we proposed a mechanism as depicted in Scheme 2 under which de-phosphorylation of ${ }^{*} N$-pSer/ Thr precursors occurs by $\beta$-elimination via a six-ring intermediate and this reaction occurs much more rapidly than proton mobilization, leading to dominant dephosphorylated molecular $\left[\mathrm{MH}_{2}-\mathrm{H}_{3} \mathrm{PO}_{4}\right]^{2+}$ and b- $\mathrm{H}_{3} \mathrm{PO}_{4}$ ions in MS/MS spectra. Thus, the $\mathrm{b}$ ions formed by backbone fragmentation following the molecular ion dephosphorylation are also dephosphorylated. However, the resulting unsaturated covalent bond on $C_{\alpha}$ of the $N$-terminus amino acid of ${ }^{*} N$-pSer/Thr precursor could suppress the formation of $\mathrm{a}_{1}$ ions since the dimethyl-vinylidene-ammonium cation is very unstable. Thus, no enhanced $a_{1}$ ion was observed for ${ }^{*} N$-pSer/Thr peptides.

Our explanations are consistent with a recent report [28] in which the magnitude of the neutral loss of phosphoric acid was found to be highly dependent on the proton mobility of the precursor ion for both phosphoserine- and phosphothreonine-containing peptides. In this study [28], peptides with internal phosphoryla- 

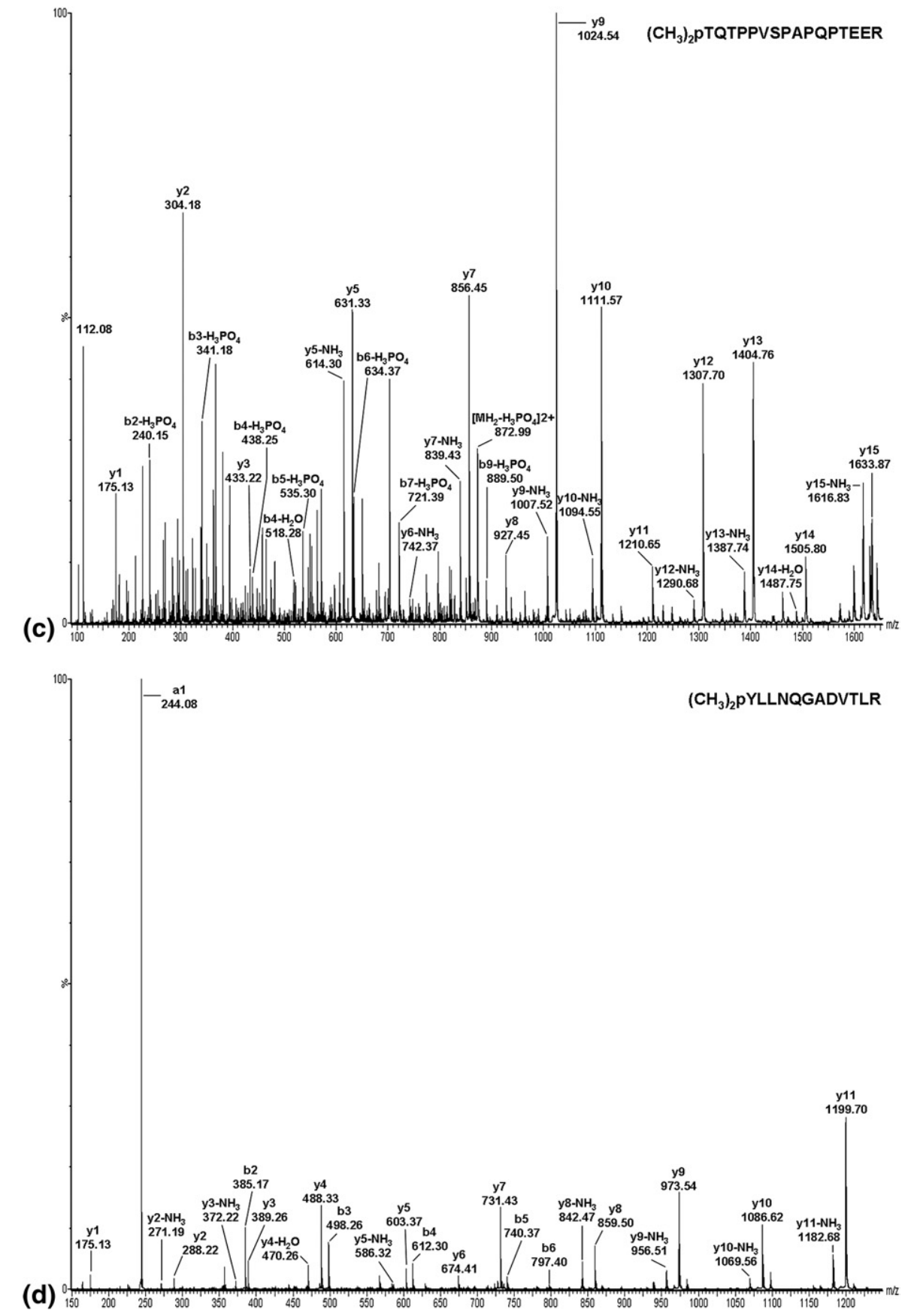

Figure 3. Continued.

tion sites were examined, and it was claimed that a charge-remote $\beta$-elimination reaction does not predominantly occur. In our study, however, peptides with $N$-terminus phosphorylation sites were examined and the dephosphorylation was not through charge-remote mechanism since the $N$-terminus charge was spatially close to the elimination sites. On the other hand, since dephosphorylation by $\beta$-elimination will not occur for peptides containing phosphotyrosine, the ${ }^{*} N$-pTyr peptides investigated here did not exhibit enhance dephosphorylation, and suppressed $\mathrm{a}_{1}$ ions as predicted based on the above mechanisms.

\section{Mapping and Quantifying Phosphorylation Sites In Vivo}

As a proof of concept, from an unfractionated A431 cell lysate, we found around $15 \%-20 \%$ of identified phosphopeptides containing or potentially containing $\mathrm{N}$ terminus phosphorylation sites. Many of them contain multiple potential phosphorylation sites near the $N$ terminal region. These sites, however, cannot be unambiguously confirmed based on either database searches or de novo sequencing. Stable isotope dimethyl labeling was performed to quantify the phosphorylation level 


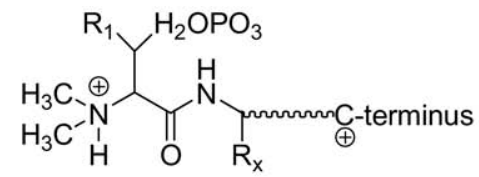

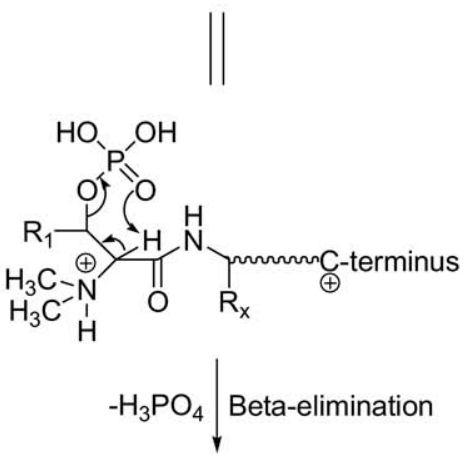

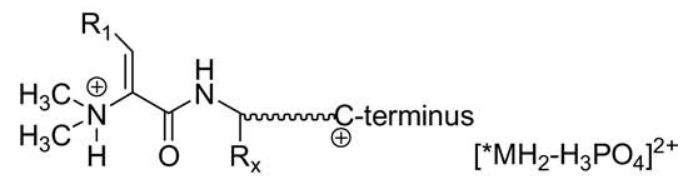
mobile proton backbone fragmentation
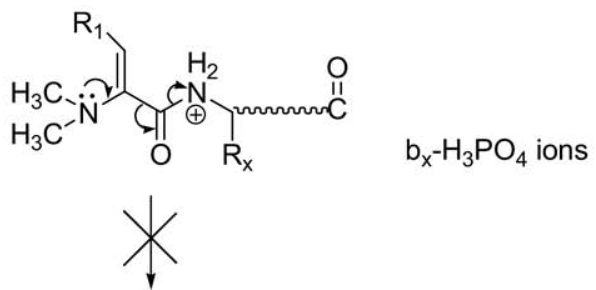

no $a_{1}$

$$
\left(\begin{array}{l}
\mathrm{H}_{3} \mathrm{C} 、 \stackrel{\oplus}{\mathrm{N}}=\mathrm{C}=\mathrm{C}^{\prime}-\mathrm{R}_{1} \\
\mathrm{H}_{3} \mathrm{C}^{-}
\end{array}\right) \text {Unstable }
$$

dimethyl-vinylidene-ammonium cation

$\mathrm{R}_{1}=\mathrm{H}$ (Ser) or $\mathrm{CH}_{3}$ (Thr)

Scheme 2. Fragmentation pathway for dimethylated phosphopeptides with phosphorylation sites at the $N$-terminus ( ${ }^{*} N$-pSer/Thr).

without $\left(H_{4}\right)$ and with $\left(D_{4}\right)$ EGF treatment. The MS/MS spectra of three phosphopeptides derived from RasGTPase-activating protein-binding protein 1 (G3BP1), $\alpha-1$ catenin (CTN1), and Src substrate cortactin (SRC8), respectively, along with their corresponding chromatograms containing the $\left(D_{4} / H_{4}\right)$ isotopic pair are shown in Figure 4. Apparently, all three MS/MS spectra show more intact molecular ions $\left[\mathrm{MH}_{2}\right]^{2+}$ than the dephosphorylated molecular ion $\left[\mathrm{MH}_{2}-\mathrm{H}_{3} \mathrm{PO}_{4}\right]^{2+}$, and all three MS/MS spectra exhibit enhanced $\mathrm{a}_{1}$ ions (Figure $4 a, b$, and c). Thus, the $N$-terminus phosphorylation site can be excluded for all three phosphopeptides, confirming the internal phosphorylation sites near the $\mathrm{N}$ terminal region: pSer 232 for Ras-GTPase-activating protein-binding protein 1, pThr401 for Src substrate cortactin, and pSer 641 for $\alpha-1$ catenin. Among these excluded sites, the site on Ser230 of G3BP1 and the site on Thr399 of SRC8 proteins were assigned to be phosphorylation sites in previous reports $[29,30]$ without confirmation. It is worth noting that the synthetic peptides shown in Figures $2 \mathrm{~b}$ and $3 \mathrm{~b}$ without and with dimethyl labeling, respectively, were the same sequence (SSPSPAPADIAQTVQEDLR) derived from Ras-GTPase-activating protein-binding protein 1 as shown in Figure $4 a$, whereas, unlike those detected from the synthetic peptides, no $\mathrm{y}_{16}$ or $\mathrm{y}_{17}$ ions (Figure 4a) could be detected from A431 cells to help in identifying the exact phosphorylation site. Likewise, the synthetic peptide shown in Figure 3c is the same sequence (TQpTPPVSPAPQPTEER) derived from Src substrate cortactin as that displayed in Figure $4 \mathrm{~b}$ but with different phosphorylation sites. Phosphorylation at the $\mathrm{N}$-terminus (Figure $3 \mathrm{c}$ ) leads to suppressed $\mathrm{a}_{1}$ ion and enhanced dephosphorylated molecular and $\mathrm{b}-\mathrm{H}_{3} \mathrm{PO}_{4}$ ions compared with that with phosphorylation at the third threonine (Figure 4b). From the corresponding chromatograms shown in Figure 4, the phosphorylation levels of these confirmed phosphorylation sites were found to slightly increase by a factor of 1.43 for TQpTPPVpSPAPQPTEER, 1.42 for TPEELDDPSDFETEDFDVR, and 1.23 for SSpSPAPADIAQTVQEDLR upon EGF stimulation. The ratio of TQpTPPVpSPAPQPTEER was further confirmed by reversed labeling as shown in Figure $4 \mathrm{~b}$. Furthermore, from unpublished data we obtained from a previous experiment performed on pregnant rat uterus homogenate [21], we confirmed a $N$ terminus phosphorylation site (pSer 43) for the pSAEDLTEGSYDAILSAEQLSAEQLEK peptide derived from similar SVH protein (Figure 4d) based on the suppressed $\mathrm{a}_{1}$ ion and the dominant dephosphorylated molecular ion $\left[\mathrm{MH}_{2}-\mathrm{H}_{3} \mathrm{PO}_{4}\right]^{2+}$ in its MS/MS spectra. The phosphorylation level of pSer 43 was shown to decrease by a factor of 0.79 upon cAMP treatment (Figure 4d). Although this $\mathrm{N}$-terminus phosphorylation site can also be identified by the dephosphorylated $b$ ions, the $a_{1}$ ion suppression provides a higher confidence and avoids misassignment due to isobaric mass effects. Notably, Ser43 is a novel phosphorylation site for the SVH protein. SVH is a novel Armadillo repeat protein that has not been reported or implied with any phosphorylation event. Our data show that the pSer 43 of SVH protein is an intrinsic phosphorylation site in pregnant rat uteri.

\section{Conclusion}

We have demonstrated that dimethyl labeling on peptide $\mathrm{N}$-terminus will enhance the dephosphorylation of the molecular ion and suppress the $a_{1}$ ion formation for Ser/Thr phosphorylated peptides with phosphorylation site at $\mathrm{N}$-terminus but not for those with phosphorylation site at the internal residues. The involved 


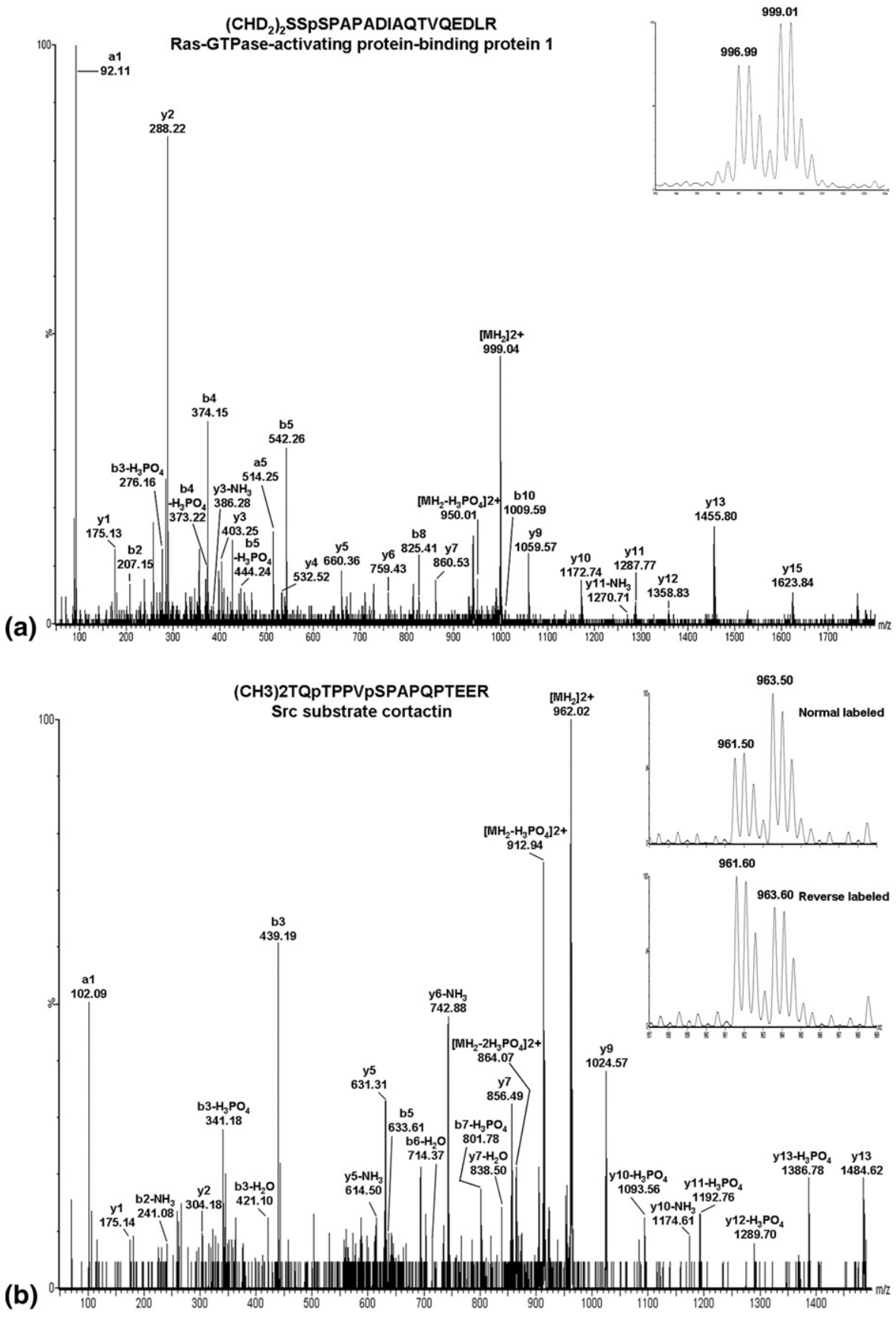

Figure 4. MS/MS spectra of dimethylated phosphopeptides derived from (a) Ras-GTPase-activating protein-binding protein 1 in A431 cells; (b) Src substrate cortactin in A431 cells, and (c) $\alpha-1$ catenin in A431 cells, (d) SVH protein in pregnant rat uterus tissue. The indents are the corresponding chromatograms of the isotopic pairs for quantification upon (a), (b), (c) EGF treatment, and (d) c-AMP treatment. For (b), Src substrate cortactin protein, the chromatogram resulting from reversed labeling was also shown to confirm the quantification ratio.

mechanisms were investigated, and a charge-remote $\beta$-elimination and mobile proton theory were proposed to explain the observations. We have then further demonstrated that the phosphorylation level and the exact phosphorylation site near $N$-terminal region can be determined simultaneously for phosphoproteins by stable isotope dimethyl labeling. Using a global analysis on A431 cells and uterus tissues as examples, we excluded the phosphorylation on Ser230 of G3BP1 and on Thr399 of SRC8 proteins in A431 cells, which were previously assigned as phosphorylation sites. We have also confirmed a known phosphorylation site on Ser634 of the CTN1 protein. The EGF-induced phosphorylation on these sites was also quantified by stable isotope dimethyl labeling. Furthermore, based on the attenuation of the $a_{1}$ ion of dimethylated peptides, we identi- 


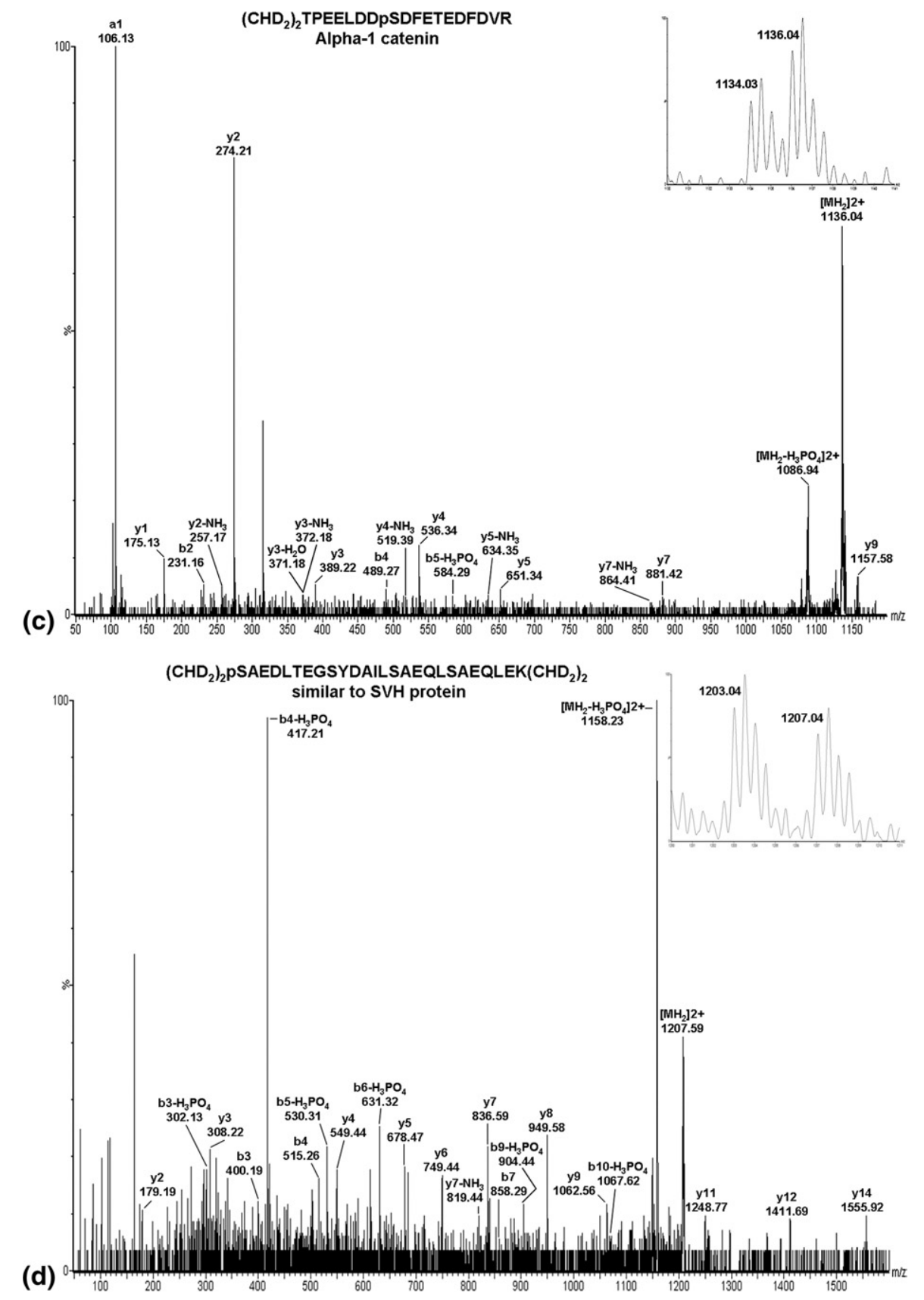

Figure 4. Continued.

fied a novel phosphorylation site on Ser43 residue at the $N$-terminus of the tryptic peptide derived from SVH protein in pregnant rat uteri. We concluded that stable isotope dimethyl labeling on peptides bears many interesting chemistries that could lead to multiple useful applications in proteomics analysis.

\section{Acknowledgments}

The authors thank the National Science Council of Taiwan for grant support. They also thank Professor Wen-Chang Chang and Professor Mei-Ling Tsai of the Medical College of National Cheng Kung University for preparing A431 cell lysate and tissue homogenate from late gestation rat uteri.

\section{Appendix A}

\section{Supplementary Material}

Supplementary material associated with this article may be found in the online version at doi:10.1016/ j.jasms.2009.12.001.

\section{References}

1. Hunter, T. Protein Kinases and Phosphatases: The Yin and Yang of Protein Phosphorylation and Signaling. Cell 1995, 80(2), 225-236.

2. Bishop, A. C.; Ubersax, J. A.; Petsch, D. T.; Matheos, D. P.; Gray, N. S.; Blethrow, J.; Shimizu, E.; Tsien, J. Z.; Schultz, P. G.; Rose, M. D.; Wood, J. L.; Morgan, D. O.; Shokat, K. M. A Chemical Switch for InhibitorSensitive Alleles of Any Protein Kinase. Nature 2000, 407(6802), 395-401. 
3. Shah, K.; Shokat, K. M. A Chemical Genetic Screen for Direct v-Src Substrates Reveals Ordered Assembly of a Retrograde Signaling Pathway. Chem. Biol. 2002, 9(1), 35-47.

4. Pawson, T.; Nash, P. Assembly of Cell Regulatory Systems Through Protein Interaction Domains. Science 2003, 300(5618), 445-452.

5. Fischer, W. H.; Hoeger, C. A.; Meisenhelder, J.; Hunter, T.; Craig, A. G. Determination of Phosphorylation Sites in Peptides and Proteins Employing a Volatile Edman Reagent. J. Protein Chem. 1997, 16(5), 329-334.

6. Gatti, A.; Traugh, J. A. A Two-Dimensional Peptide Gel Electrophoresis System for Phosphopeptide Mapping and Amino Acid Sequencing. Anal. Biochem. 1999, 266(2), 198-204.

7. MacDonald, J. A.; Mackey, A. J.; Pearson, W. R. Haystead, T. A. A Strategy for the Rapid Identification of Phosphorylation Sites in the Phosphoproteome. Mol. Cell. Proteom. 2002, 1(4), 314-322.

8. Meyer, H. E.; Hoffmann-Posorske, E.; Korte, H.; Heilmeyer, L. M. Jr. Sequence Analysis of Phosphoserine-Containing Peptides. Modification for Picomolar Sensitivity. FEBS Lett 1986, 204(1), 61-66.

9. Aebersold, R.; Watts, J. D.; Morrison, H. D.; Bures, E. J. Determination of the Site of Tyrosine Phosphorylation at the Low Picomole Level by Automated Solid-Phase Sequence Analysis. Anal. Biochem. 1991, 199(1), $51-60$

10. Oda, Y.; Nagasu, T.; Chait, B. T. Enrichment Analysis of Phosphorylated Proteins as a Tool for Probing the Phosphoproteome. Nat. Biotechnol. 2001, 19 (4), 379-382.

11. Ficarro, S. B.; McCleland, M. L.; Stukenberg, P. T.; Burke, D. J.; Ross, M. M.; Shabanowitz, J.; Hunt, D. F.; White, F. M. Phosphoproteome Analysis by Mass Spectrometry and Its Application to Saccharomyces cerevisiae. Nat Biotechnol 2002, 20(3), 301-305.

12. Mann, M.; Ong, S. E.; Gronborg, M.; Steen, H.; Jensen, O. N.; Pandey, A. Analysis of Protein Phosphorylation Using Mass Spectrometry: Deciphering the Phosphoproteome. Trends Biotechnol. 2002, 20(6), 261-268.

13. Steen, H.; Mann, M. A New Derivatization Strategy for the Analysis of Phosphopeptides by Precursor Ion Scanning in Positive Ion Mode. J. Am. Soc. Mass Spectrom. 2002, 13(8), 996-1003.

14. Gunawardena, H. P.; Emory, J. F.; McLuckey, S. A. Phosphopeptide Anion Characterization Via Sequential Charge Inversion and ElectronTransfer Dissociation. Anal. Chem. 2006, 78(11), 3788-3793.

15. Wiesner, J.; Premsler, T.; Sickmann, A. Application of Electron Transfer Dissociation (ETD) for the Analysis of Post-Translational Modifications. Proteomics 2008, 8(21), 4466-4483.

16. Ben Hamidane, H.; Chiappe, D.; Hartmer, R.; Vorobyev, A.; Moniatte, M.; Tsybin, Y. O. Electron Capture and Transfer Dissociation: Peptide Structure Analysis at Different Ion Internal Energy Levels. J. Am. Soc. Mass Spectrom. 2009, 20(4), 567-575.

17. Rusnak, F.; Zhou, J.; Hathaway, G. Identification of Phosphorylated and Glycosylated Sites in Peptides by Chemically Targeted Proteolysis. J. Biomol. Tech. 2002, 13(4), 228-237.
18. Knight, Z. A.; Schilling, B.; Row, R. H.; Kenski, D. M.; Gibson, B. W.; Shokat, K. M. Phosphospecific Proteolysis for Mapping Sites of Protein Phosphorylation. Nat. Biotechnol. 2003, 21(9), 1047-1054.

19. Kristensen, L. H.; Nielsen, P. E.; Jorgensen, C. I.; Kragelund, B. B.; Mollegaard, N. E. Phosphate Selective Uranyl Photo-Affinity Cleavage of Proteins. Determination of Phosphorylation Sites. Chem. Biochem. 2008, 9(15), 2377-2381.

20. Hsu, J. L.; Huang, S. Y.; Shiea, J. T.; Huang, W. Y.; Chen, S. H. Beyond Quantitative Proteomics: Signal Enhancement of the a1 Ion as a Mass Tag for Peptide Sequencing Using Dimethyl Labeling. J. Proteome Res. 2005, 4(1), 101-108.

21. Shen, P. T.; Hsu, J. L.; Chen, S. H. Dimethyl Isotope-Coded Affinity Selection for the Analysis of Free and Blocked N-Termini of Proteins using LC-MS/MS. Anal. Chem. 2007, 79(24), 9520-9530.

22. Huang, S. Y.; Tsai, M. L.; Wu, C. J.; Hsu, J. L.; Ho, S. H.; Chen, S. H. Quantitation of Protein Phosphorylation in Pregnant Rat Uteri Using Stable Isotope Dimethyl Labeling Coupled with IMAC. Proteomics 2006, 6(6), 1722-1734

23. Hsu, J. L.; Huang, S. Y.; Chow, N. H.; Chen, S. H. Stable-Isotope Dimethyl Labeling for Quantitative Proteomics. Anal. Chem. 2003, 75(24), 6843-6852.

24. Reid, G. E.; Simpson, R. J.: O'Hari, R. A. Leaving Group and Gas Phase Neighboring Group Effects in the Side Chain Losses from Protonated Serine and Its Derivatives. J. Am. Soc. Mass Spectrom. 2000, 11(12) 1047-1060.

25. Gronert, S.; Li, K. H.; Horiuchi, M. Manipulating the Fragmentation Patterns of Phosphopeptides Via Gas-Phase Boron Derivatization: Determining Phosphorylation Sites in Peptides with Multiple Serines. J. Am. Soc. Mass Spectrom. 2005, 16(12), 1905-1914.

26. Fu, Q.; Li, L. De Novo Sequencing of Neuropeptides Using Reductive Isotopic Methylation and Investigation of ESI OTOF MS/MS Fragmentation Pattern of Neuropeptides with N-Terminal Dimethylation. Anal. Chem. 2005, 77(23), 7783-7795.

27. Liu, N.; Chan, W.; Lee, K. C.; Cai, Z. A Method to Enhance $a_{1}$ Ions and Application for Peptide Sequencing and Protein Identification. J. Am Soc. Mass Spectrom. 2009, 20(6), 1214-1223.

28. Palumbo, A. M.; Tepe, J. J. Reid, G. E. Mechanistic Insights Into the Multistage Gas-Phase Fragmentation Behavior of Phosphoserine- and Phosphothreonine-Containing Peptides. J. Proteome Res. 2008, 7(2), 771779.

29. Zheng, H.; Hu, P.; Quinn, D. F.; Wang, Y. K. Phosphotyrosine Proteomic Study of Interferon $\alpha$ Signaling Pathway Using a Combination of Immunoprecipitation and Immobilized Metal Affinity Chromatography. Mol. Cell. Proteom. 2005, 4(6), 721-730.

30. Zahedi, R. P.; Lewandrowski, U.; Wiesner, J.; Wortelkamp, S.; Moebius, J.; Schutz, C.; Walter, U.; Gambaryan, S.; Sickmann, A. Phosphoproteome of Resting Human Platelets. J. Proteome Res. 2008, 7(2), 526-534. 